\title{
CLASSICAL GROUPS AND GENERALIZED SIMPLE GROUPS OF LIE TYPE
}

\author{
CHENG CHON HU
}

(Received 16 July 1987; revised 16 June 1988)

Communicated by $\mathbf{H}$. Lausch

\begin{abstract}
In this note, for any given simple group obtained from an orthogonal or unitary group of nonzero index, by a procedure similar to the construction of Chevalley groups and twisted groups, we construct a simple group which is identified with the given simple classical group. The simple groups constructed in this note can be interpreted as generalized simple groups of Lie type. Thus all simple groups of Lie type of types $A_{n}, B_{n}, C_{n}$ and $D_{n}$ and all generalized simple groups of Lie type constructed in this note exhaust all simple classical groups with non-zero indices.
\end{abstract}

1980 Mathematics subject classification (Amer. Math. Soc.) (1985 Revision): 20 H 20.

In [3], by a similar method to that used in [4], [6], [2], we constructed a family of simple groups associated with the Satake diagrams. The simple groups associated with the Satake diagrams of types AIII, BI, DI are identified with some simple groups obtained from the orthogonal or unitary groups corresponding to the forms whose Witt indices and the anisotropic kernels were not considered explicitly in [3]. In general, the simple groups of Lie type of the types $A_{n}, B_{n}, C_{n}, D_{n}$ are the linear and symplectic groups, and orthogonal and unitary groups corresponding to the forms whose Witt indices are sufficiently large. The remaining simple classical groups are not Chevalley groups or twisted groups, that is, the simple groups of Lie type. In this note, for constructing the remaining simple classical groups, we consider Witt indices and the anisotropic kernels of the forms carefully and we get a method which is similar to the method of [4], [6] and [2], and is more explicit than the

(C) 1989 Australian Mathematical Society 0263-6115/89 \$A2.00+0.00 
method of [3]. By this method, for a simple classical group corresponding to a given form, we construct a simple group which is identified with the simple classical group. The simple groups constructed in this note can be interpreted as generalized simple groups of Lie type. It follows that all simple groups of Lie type of types $A_{n}, B_{n}, C_{n}$ and $D_{n}$ and all generalized simple groups of Lie type constructed in this note exhaust all simple classical groups with non-zero indices. Furthermore, in this note, we prove that every generalized simple group of Lie type has a "quasi-Bruhat" decomposition which is a generalization of the Bruhat decomposition of the simple groups of Lie type and has a $(B, N)$ pair. Thus, in this note, we prove that every simple classical group has a "quasi-Bruhat" decomposition and has a $(B, N)$ pair also.

Let $K$ be a field of characteristic not equal to 2 .

Now, we shall consider the Witt index and the anisotropic kernel of a Hermitian form or of a quadratic form.

(I) Let $K$ be a field such that there is a non-trivial involutive automorphism $\sigma$ of $K$. For each $t \in K$, we write $\sigma(t)=\bar{t}$. Let $V$ be a vector space of dimension $n+1$ over $K$ endowed with a non-singular Hermitian scalar product which determines a Hermitian form $f$. We denote by $\nu$ (or $\nu(f))$ the Witt index of $f$. Then the Hermitian form $f$ relative to the basis $B=\left\{v_{0}, \ldots, v_{n}\right\}$ has the form:

$$
\begin{gathered}
f=\bar{x}_{0} x_{n}+\bar{x}_{n} x_{0}+\bar{x}_{1} x_{n-1}+\bar{x}_{n-1} x_{1}+\cdots+\bar{x}_{\nu-1} x_{n-\nu+1}+\bar{x}_{n-\nu+1} x_{\nu-1} \\
+\gamma_{1} \bar{x}_{\nu} x_{\nu}+\gamma_{2} \bar{x}_{\nu+1} x_{\nu+1}+\cdots+\gamma_{k+1} \bar{x}_{\nu+k} x_{\nu+k}, \\
k=n-2 \nu, \quad \gamma_{i} \in K, \quad \sigma\left(\gamma_{i}\right)=\gamma_{i}, \quad i=1, \ldots, k+1 .
\end{gathered}
$$

It is clear that $\left(\gamma_{1}, \gamma_{2}, \ldots, \gamma_{k+1}\right)$, denoted by $\Delta_{0}(f)$, is associated with the anisotropic kernel of the form $f$. Clearly, we have $\sum_{i=1}^{k+1} \gamma_{i} t_{i} \bar{t}_{i} \neq 0$ for all $\left(t_{1}, t_{2}, \ldots, t_{k+1}\right) \in K^{(k+1)},\left(t_{1}, t_{2}, \ldots, t_{k+1}\right) \neq 0$.

(II) Let $V$ be a vector space of dimension $n+1$ over $K$ endowed with a non-singular symmetric scalar product which determines a quadratic form $f$. We denote by $\nu$ (or $\nu(f)$ ) the Witt index of $f$. Then the quadratic form $f$ relative to the basis $B=\left\{v_{0}, \ldots, v_{n}\right\}$ has the form

$$
\begin{gathered}
f=x_{0} x_{n}+x_{1} x_{n-1}+\cdots+x_{\nu-1} x_{n-\nu+1}+\gamma_{1} x_{\nu}^{2}+\gamma_{2} x_{\nu+1}^{2}+\cdots+\gamma_{k+1} x_{\nu+k}^{2}, \\
k=n-2 \nu, \quad \gamma_{i} \in K, \quad i=1, \ldots, k+1 .
\end{gathered}
$$

It is clear that $\left(\gamma_{1}, \gamma_{2}, \ldots, \gamma_{k+1}\right)$, denoted by $\Delta_{0}(f)$, is associated with the anisotropic kernel of the form $f$. Clearly, we have $\sum_{i=1}^{k+1} \gamma_{i} t_{i}^{2} \neq 0$ for all $\left(t_{1}, t_{2}, \ldots, t_{k+1}\right) \in K^{(k+1)},\left(t_{1}, t_{2}, \ldots, t_{k+1}\right) \neq 0$.

We will use the notations defined in [1] without explanation.

Henceforth, we fix a form $f$ which is a Hermitian form of a quadratic form and we denote by $\nu$ the Witt index $\nu(f)$ of $f$. 
Let $B=\left\{v_{0}, v_{1}, \ldots, v_{n}\right\}$ be a basis of vector space $V_{C}$ of dimension $n+1$ over C. Relative to the basis $B$, each linear transformation of $V_{C}$ can be expressed as an $(n+1) \times(n+1)$ matrix over $C$. Let $L$ be the Lie algebra of all $(n+1) \times(n+1)$ matrices of trace zero over $\mathbb{C}$. Then $L \cong A_{n}$. Let $e_{i j}$ be the elementary matrix with $(i, j)$-coefficient 1 and other coefficients 0 and $h_{i j}=e_{i i}-e_{j j}, 0 \leq i, j \leq n, i \neq j$. Let $h$ be the subalgebra of all diagonal matrices in $L$. It is clear that $L=h+\sum_{i \neq j} C e_{i j}$ is a root space decomposition of $L$ where $C e_{i j}$ is the root space of $L$ corresponding to the co-root $h_{i j}, 0 \leq i, j \leq n, i \neq j$. Clearly, $h$ is a Cartan subalgebra of $L$ and $h$ is a subspace of $L$ spanned by $\left\{h_{i j}, 0 \leq i, j \leq n, i \neq j\right\}$ over $\mathbb{C}$.

Let $B_{0}=\left\{e_{0}, e_{1}, \ldots, e_{n}\right\}$ be an orthonormal basis of a Euclidean space of dimension $n+1$. It is convenient that we denote by $e_{i}-e_{j}$ the root corresponding to the co-root $h_{i j}, 0 \leq i, j \leq n, i \neq j$. Thus, the root system $\Phi$ of $L$ relative to $h$ can be expressed in the form $\left\{e_{i}-e_{j}, 0 \leq i, j \leq n, i \neq j\right\}$ and $\Delta=\left\{e_{i}-e_{i+1}, 0 \leq i \leq n-1\right\}$ is a fundamental root system of $\Phi$.

Let $\Theta$ be the map of $B_{0}$ defined by $\Theta\left(e_{i}\right)=-e_{n-i}, \Theta\left(e_{n-i}\right)=-e_{i}, 0 \leq i \leq$ $\nu-1, \Theta\left(e_{j}\right)=-e_{j}, \nu \leq j \leq n-\nu$. Clearly, $\Theta$ can be extended as a linear transformation of $h$ which is denoted by $\boldsymbol{\theta}$ also, and $\boldsymbol{\theta}=\tau w_{0}$ where $\tau$ is a non-trivial symmetry of the Dynkin diagram of $\Delta$ and $w_{0}$ is an element of the Weyl group of $\Phi$. For each $r \in \Phi$, we define $\bar{r}=\boldsymbol{\theta}(r)$ and $r^{\prime}=\frac{1}{2}(r+\bar{r})$. Let $\Delta_{0}=\left\{r \in \Delta ; r^{\prime}=0\right\}$ and $\Phi_{0}=\left\{r \in \Phi ; r^{\prime}=0\right\}$. A Dynkin diagram of $\Delta$ is called the diagram of form $f$ if every root of $\Delta_{0}$ is denoted by a black node and every root of $\Delta^{*}=\Delta \backslash \Delta_{0}$ is denoted by a white node, and two distinct roots $r_{1}$ and $r_{2}$ of $\Delta^{*}$ are joined by a curved arrow when $r_{1}^{\prime}=r_{2}^{\prime}$. Clearly, the linear transformation $\boldsymbol{\theta}$ of $h$ and the diagram of form $f$ which is identified with the Satake diagram of type All given in Table I are determined by $\nu$. We define $\boldsymbol{\Phi}^{*}=\boldsymbol{\Phi} \backslash \boldsymbol{\Phi}_{0} ; \boldsymbol{\Phi}_{1}=\left\{r \in \boldsymbol{\Phi}^{*} ; r=\tilde{F}\right\} ; \boldsymbol{\Phi}_{11}=\boldsymbol{\Phi}^{*} \backslash \boldsymbol{\Phi}_{1} ; \boldsymbol{\Phi}_{11 b}=\left\{r \in \boldsymbol{\Phi}_{11}\right.$; $r+\boldsymbol{r} \in \boldsymbol{\Phi}\} ; \boldsymbol{\Phi}_{11 a}=\boldsymbol{\Phi}_{11} \backslash \boldsymbol{\Phi}_{11 b} ; \boldsymbol{\Phi}_{1 b}=\left\{r+\bar{r} ; r \in \boldsymbol{\Phi}_{11 b}\right\} ; \boldsymbol{\Phi}_{1 a}=\boldsymbol{\Phi}_{1} \backslash \boldsymbol{\Phi}_{1 b}$.

For $\Delta_{0}(f)=\left\{\gamma_{1}, \gamma_{2}, \ldots, \gamma_{k+1}\right\}, k=n-2 \nu$, we define

$$
A_{0}=\operatorname{diag}\left(\gamma_{1}, \gamma_{2}, \ldots, \gamma_{k+1}\right) \text {. }
$$

Let $J_{\nu}$ be the $\nu \times \nu$ matrix

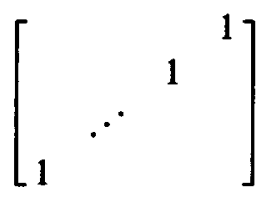

and let $A$ be the matrix

$$
\left[\begin{array}{lll} 
& & J_{\nu} \\
& A_{0} & \\
J_{\nu} & &
\end{array}\right] .
$$


Clearly, $C_{b}\left\{h_{i i+1}, 0 \leq i \leq n-1 ; e_{i j}, 0 \leq i, j \leq n, i \neq j\right\}$ is a Chevalley basis of $L$. Let $L_{Z}$ be the subset of all linear combinations of the elements of $C_{b}$ with the coefficients in $Z$ of rational integers and $L_{K}=L_{Z} \otimes K$ (see page 62 of [1]). We define a transformation $\rho_{\Theta}$ of $L_{K}$ by $\rho_{\Theta}(M)=-A M^{\prime} A^{-1}$, for all $M \in L_{K}$. Clearly, $\rho_{\Theta}$ is an automorphism of $L_{K}$. Since $A^{\prime} A^{-1}=I$ we have $\rho_{\Theta}^{2}(M)=M$ for all $M \in L_{K}$, so $\rho_{\Theta}^{2}=I$ where $I$ is the identity map.

Clearly, $\Phi_{11 b}=\left\{ \pm\left(e_{i}-e_{j}\right), i=0, \ldots, \nu-1, \nu+k+1, \ldots, n, j=\nu, \ldots, \nu+k\right\}$ and $\Phi_{1} \cup \Phi_{11 a}=\left\{ \pm\left(e_{i}-e_{j}\right), i, j=0, \ldots, \nu-1, \nu+k+1, \ldots, n, i<j\right\}$, so we have $\rho_{\Theta}\left(e_{r}\right)=-k_{r} e_{r}, r \in \Phi, e_{r}, e_{r} \in C_{b}$ where $K_{r} \in K$ satisfy the condition (A): AI, $k_{r}=1$ if $r \in \Phi_{11 a} \cup \Phi_{1} ; \gamma_{i}=\gamma_{j}^{-\lambda}$ if $r=\lambda\left(e_{i}-e_{j}\right) \in \Phi_{11 b}$, $j=\nu, \ldots, \nu+k, i=0,1, \ldots, \nu-1, \nu+k+1, \ldots, n, \lambda=1$ or -1 ; AII, $k_{r} k_{r}=1$ and $k_{r} k_{-r}=1$ for all $r \in \Phi$.

We may summarize the results mentioned above as follows.

Proposition 1. Let $f$ be a Hermitian form or quadratic form such that $\nu(f)=\nu$ and $\Delta_{0}(f)=\left(\gamma_{1}, \gamma_{2}, \ldots, \gamma_{k+1}\right)$. Then there exist a Chevalley basis $C_{b}=\left\{h_{r}, r \in \Delta ; e_{r}, r \in \Phi\right\}$ of $L=A_{n}, n=2 \nu+k$ and an involutive automorphism $\rho_{\Theta}$ of $L_{K}=L_{Z} \otimes K$ such that $\rho_{\Theta}\left(e_{r}\right)=-k_{r} e_{-r}, r \in \Phi, e_{r}, e_{r} \in C_{b}$ where $k_{r} \in K$ satisfy the condition (A) mentioned above.

Henceforth, we denote by $F$ the non-trivial involutive automorphism $\sigma$ of $K$ if $f$ is a Hermitian form, we denote by $F$ the identity transformation $I$ of $K$ if $f$ is a quadratic form, and we write $F(t)=\bar{t}$ for any $t \in K$. We denote by $G$ the Chevalley group $L(K)=\left\langle x_{r}(t)=\exp \left(\operatorname{tade}_{r}\right) ; r \in \Phi, t \in K\right)$. Clearly, $F$ can be extended to an involutive automorphism $F$ of $G$ in this way: $F\left(x_{r}(t)\right)=x_{r}(\bar{t}), r \in \Phi, t \in K$, Moreover, the involutive automorphism $\rho_{\Theta}$ of $L_{K}$ can be extended to an involutive automorphism of $G$ (denoted by $\rho_{\Theta}$ also) in this way: $\rho_{\Theta}\left(x_{r}(t)\right)=x_{r}\left(-k_{r} t\right), r \in \Phi, t \in K$. We define an automorphism $\sigma$ of $G$ by $\sigma=\rho_{\Theta} F$, so $\sigma\left(x_{r}(t)\right)=x_{r}\left(-k_{r} \bar{t}\right), r \in \Phi, t \in K$ because $k_{r}=k_{r}$ for all $r \in \Phi$. Obviously, we have $\rho_{\Theta}^{2}=I$ and $F^{2}=I$ and $\rho_{\Theta} F=F \rho_{\Theta}$, so $\sigma^{2}=I$. Thus $\sigma$ is an involutive automorphism of $G$.

We define some notation and terminology which will be used later.

(I) If $r \in \Phi_{1}, X_{r}^{!}(t)=x_{r}(t), t \in K_{r}=\{t \in K ; \bar{t}=-t\}, W_{r}^{1}=w_{r} ; h_{r}^{1}(v)=$ $h_{r}(v), v \in K_{r}^{* \prime}=\{v \in K ; v=v\} ; N_{r}^{1}(u)=n_{r}(u), u \in K_{r}^{*}=K_{r} \backslash 0$.

(IIa) If $r \in \Phi_{11 a}, X_{r}^{1}(t)=x_{r}(t) x_{r}(-\bar{t}), t \in K_{r}=K$; $W_{r}^{1}=w_{r} w_{r} ; h_{r}^{1}(v)=$ $h_{r}(v) h_{r}(v), v \in K_{r}^{* \prime}=K_{r} \backslash 0 ; N_{r}^{1}(u)=n_{r}(u) n_{r}(-\bar{u}), u \in K_{r}^{*}=K_{r} \backslash 0$.

(IIb) If $r \in \Phi_{11 b}, X_{r}^{1}(t)=x_{r}(t) x_{r}\left(-k_{r} \bar{t}\right) x_{r+r}\left(-\frac{1}{2} N_{r, r} k_{r}(t \bar{t}), t \in K_{r}=K\right.$; $W_{r}^{1}=w_{r+r}, h_{r}^{1}(v)=h_{r}(v) h_{r}(v), v \in K_{r}^{* \prime}=K_{r} \backslash 0 ; N_{r}^{1}(u)=h_{r}(u) n_{r} n_{r} n_{r}$, $u \in K_{r}^{* 1}$.

For each $r \in \Phi^{*}$, we write $r^{\prime}=\frac{1}{2}(r+\bar{r})$ and define $I_{1}(r)=\{s \in I(r) ; s \geq s\}$, where $I(r)=\left\{s \in \Phi^{*} ; s^{\prime}=\eta r^{\prime}, \eta=1, \frac{1}{2}\right.$ or 2$\}$. 
The following statement $(B)$ is easily verified.

(BI) If $r=\lambda\left(e_{j}-e_{q}\right), j=0,1, \ldots, \nu-1, q=n-j, r \in \Phi_{1 b}, \lambda=1$ or -1 ,

$$
\begin{aligned}
I_{1}(r)=\left\{r_{i}=\lambda\left(e_{j}-e_{\nu-1+i}\right), i\right. & \left.=1, \ldots, k+1 ; r=r_{p}, p=k+1+1\right\}, \\
I(r)=\left\{r_{i}=\lambda\left(e_{j}-e_{\nu-1+i}\right), r_{i}\right. & =\lambda\left(e_{\nu-1+i}-e_{q}\right), \\
i & \left.=1, \ldots, k+1 ; r=r_{p}, p=k+1+1\right\} .
\end{aligned}
$$

(BIIa) If $r \in \Phi_{11 a}, I_{1}(r)=\left\{r_{p}\right\}, r_{p}=r$ or $\bar{r}$ according to $r \geq \bar{r}$ or $\bar{r}>r, p=$ $1, I(r)=\{r, r\}(r \neq \dot{r})$.

(BIIb) If $r \in \Phi_{11 b}, I_{1}(r)=I_{1}(r+\bar{r}), I(r)=I(r+\bar{r}), r+\bar{r} \in \Phi_{1 b}$.

For any $r \in \Phi^{*}$, we define $K_{(r)}=K_{r_{1}} \times \cdots \times K_{r_{p}}, X_{r}(T)=\prod_{i=1}^{p} X_{r_{i}}^{1}\left(t_{i}\right)$, $T=\left(t_{1}, t_{2}, \ldots, t_{p}\right) \in K_{(r)}$, and $D(T)=\sum_{i=1}^{k+1} \gamma_{i} \bar{t}_{i} t_{i}$. We write $N_{r}^{1}=n_{r} n_{r}$ if $r \in \Phi_{11 a}, N_{r}^{1}=n_{r} n_{r} n_{r}$ if $r \in \Phi_{11 b}, N_{r}^{1}=n_{r}\left(u_{0}\right), u_{0}$ being a fixed element of $K_{r}^{*}$ if $r \in \Phi_{1}$ and $K_{r}^{*} \neq 0$. We define $W^{1}=\left\langle w_{r}^{1}, r \in \Phi^{*}\right\rangle, N^{1}=\left\langle N_{r}^{1}(u), r \in \Phi^{*}\right.$, $\left.u \in K_{r}^{*}\right\rangle_{i} H^{1}=N^{1} \cap H ; U^{1}=\{u \in U ; \sigma(u)=u\}, V^{1}=\{v \in V ; \sigma(v)=v\}$, $G^{1}=\left\langle U^{1}, V^{1}\right\rangle, G_{0}=\left\langle x_{\alpha}(t), \alpha \in \Phi_{0}, t \in K ; H\right\rangle, Y^{1}=G_{0} \cap G^{1}$.

Clearly, for any form $f$ given by (I) or (II), the group $G^{1}$ is determined by $\nu(f), \Delta_{0}(f)$ and $K, F$, so we denote by $A_{n}\left(\nu(f), \Delta_{0}(f) ; K, F\right)$ the group $G^{1}$.

We denote by $\Phi^{+}$(respectively $\Phi^{-}$) the positive (respectively negative) root system containing $\Delta$ (respectively $-\Delta$ ), clearly, we have $\Phi^{+}=-\Phi^{-}$.

We define $\boldsymbol{\Phi}^{*+}=\Phi^{*} \cap \Phi^{+}$and $\Phi^{*-}=-\Phi^{*+}, \Sigma_{I}^{*+}=\left\{r \in \Phi^{*+} ; r \geq s\right.$, $s \in I(r)\}$ and $\Sigma_{I}^{*-}=-\Sigma_{I}^{*+}, \Delta_{I}^{*}=\left\{r \in \Delta^{*} ; r \geq \bar{r}\right\}$ and $\Phi_{I}^{*+}=\left\{r \in \Phi^{*+} ; r \geq \bar{r}\right\}$.

Let $W_{0}=\left\langle w_{\alpha}, \alpha \in \Phi_{0}\right\rangle$ and $W_{0}^{1}=W_{0} \cap W^{1}$.

By [3] we have the following propositions and corollary immediately.

Proposition 2. (a) $W^{1}=\left\langle W_{r}^{1}, r \in \Delta_{I}^{*} ; W_{0}^{1}\right\rangle$.

(b) Let $r_{1}, s \in \Phi^{*}$. Then there exists $w \in W^{1}$ such that $\left(s_{1}^{\prime}, r_{1}^{\prime}\right) \neq 0, s_{1}=$ $w(s)$.

(c) Let $s \in \Phi^{*}$. Then there exist $w \in W^{1}$ and $r \in \Delta_{I}^{*}$ such that $w(s) \in I(r)$.

Proposition 3. (a) Let $r \in \Phi_{I}^{*+}, s \in \Sigma_{I}^{*+}, t \in K_{r}^{*}$ and $T \in K_{(s)}$. Then $X_{r}^{1}(t), X_{s}(T) \in U^{1}$ and $X_{-r}^{1}(t), X_{-s}(T) \in V^{1}$.

(b) Each element $u$ of $U^{1}(u \neq I)$ and each element $v$ of $V^{1}(v \neq I)$ have a unique expression in the forms

$$
\begin{aligned}
& u=\prod X_{r_{i}}^{1}\left(t_{i}\right)=\prod X_{s_{j}}\left(T_{j}\right), \\
& r_{i} \in \Phi_{I}^{*+}, s_{j} \in \Sigma_{l}^{*+}, t_{i} \in K_{r_{i}}^{*}, T_{j} \in K_{\left(s_{j}\right)}, T_{j} \neq(0, \ldots, 0)=0, \\
& i=1,2, \ldots, p, j=1,2, \ldots, q, r_{1}<r_{2}<\cdots<r_{p}, s_{1}<s_{2}<\cdots<s_{q} . \\
& v=\prod X_{-r_{i}}^{1}\left(t_{i}\right)=\prod X_{-s_{j}}\left(T_{j}\right), \\
& \quad r_{i} \in \Phi_{I}^{*+}, s_{j} \in \Sigma_{I}^{*+}, t_{i} \in K_{r_{i}}^{*}, T_{j} \in K_{\left(s_{j}\right)}, T_{j} \neq 0, i=1,2, \ldots, l, \\
& \quad j=1,2, \ldots, m, r_{1}<r_{2}<\cdots<r_{l}, s_{1}<s_{2}<\cdots<s_{m} .
\end{aligned}
$$


We shall aefine $J_{1}(u)=\left\{r_{1}\right\}, J_{1}(v)=\left\{-r_{1}\right\} ; J(u)=\left\{r_{1}, \ldots, r_{p}\right\} ; J(v)=$ $\left\{-r_{1}, \ldots,-r_{l}\right\}, J(u)^{*}=\left\{s_{1}, \ldots, s_{q}\right\} ; J(v)^{*}=\left\{-s_{1}, \ldots,-s_{m}\right\}$.

Corollary 1. Let $r \in \Delta_{I}^{*}$ and $U, T \in K_{(r)}$. Let $u^{\prime}=N_{r}^{1} u\left(N_{r}^{1}\right)^{-1}$ and $u^{\prime \prime}=X_{-r}(U) u X_{-r}(-U)$, and $v^{\prime}=N_{r}^{1} v\left(N_{r}^{1}\right)^{-1}$ and $v^{\prime \prime}=X_{r}(T) v X_{r}(-T)$ where $u \in U^{1}$ and $v \in V^{1}$ satisfy $r \notin J(u)^{*}$ and $-r \notin J(v)^{*}$. Then $u^{\prime}, u^{\prime \prime} \in U^{1}$ and $v^{\prime}, v^{\prime \prime} \in V^{\prime}$ satisfy $r \notin J\left(u^{\prime}\right)^{*} \cup J\left(u^{\prime \prime}\right)^{*}$ and $-r \notin J\left(v^{\prime}\right)^{*} \cup J\left(v^{\prime \prime}\right)^{*}$.

For each $r \in \Phi_{11 b}$, we write $s=r+\bar{r}$ and $\eta=N_{r, r}$. By 6.4 .4 and 5.2 .2 of [1], and $\eta^{2}=1$, for each $t \in K_{r}^{*}$, we have

$$
\begin{aligned}
X_{r}^{1}(t)= & x_{r}(t) x_{r}\left(-k_{r} \bar{t}\right) x_{s}\left(\frac{1}{2} \eta k_{r} t \bar{t}\right) \\
= & x_{-r}\left(t^{-1}\right) h_{-r}\left(-t^{-1}\right) n_{r} x_{r}\left(-k_{r} \bar{t}\right) x_{r}\left(\eta \frac{1}{2} \eta k_{r} t \bar{t} t^{-1}\right) x_{s}\left(\frac{1}{2} \eta k_{r} t \bar{t}\right) x_{-r}\left(t^{-1}\right) \\
= & x_{-r}\left(t^{-1}\right) h_{-r}\left(-t^{-1}\right) n_{r} x_{r}\left(-\frac{1}{2} k_{r} \bar{t}\right) x_{r}\left(\frac{1}{2} \eta k_{r} t \bar{t}\right) x_{-r}\left(t^{-1}\right) \\
= & x_{-r}\left(t^{-1}\right) h_{-r}\left(-t^{-1}\right) x_{-s}\left(2 \eta k_{r}^{-1} \bar{t}^{-1}\right) h_{-s}\left(2 k_{r}^{-1} \bar{t}^{-1}\right) \\
& n_{r} n_{r} x_{r}(t) x_{s}\left(\frac{1}{2} \eta k_{r} t \bar{t}\right) x_{-r}\left(2 k_{r}^{-1} \bar{t}^{-1}\right) x_{-r}\left(t^{-1}\right) \\
= & x_{-r}\left(t^{-1}\right) h_{-r}\left(-t^{-1}\right) x_{-s}\left(2 \eta k_{r}^{-1} \bar{t}^{-1}\right) h_{-s}\left(2 k_{r}^{-1} \bar{t}^{-1}\right) \\
& x_{-r}\left(t^{-1}\right) h_{-r}\left(t^{-1}\right) n_{r} n_{r} n_{r} \\
& x_{r}\left(-\frac{1}{2} k_{r} \bar{t}\right) x_{s}\left(\frac{1}{2} \eta k_{r} t \bar{t}\right) x_{-r}\left(t^{-1}\right) x_{-r}\left(2 k_{r}^{-1} \bar{t}^{-1}\right) x_{-r}\left(t^{-1}\right) \\
= & x_{-r}\left(t^{-1}\right) h_{-r}\left(-t^{-1}\right) x_{-s}\left(2 \eta k_{r}^{-1} \bar{t}^{-1}\right) h_{-s}\left(2 k_{r}^{-1} \bar{t}^{-1}\right) \\
& x_{-r}\left(t^{-1}\right) h_{-r}\left(t^{-1}\right) x_{-r}\left(-\frac{1}{2} k_{r} \bar{t}\right) \\
& x_{-s}\left(\frac{1}{2} \eta k_{r} t \bar{t}\right) n_{r} n_{r} n_{r} x_{-r}\left(2 t^{-1}\right) x_{-r}\left(2 k_{r}^{-1} \bar{t}^{-1}\right) x_{-s}\left(2 \eta k_{r}^{-1} t^{-1} \bar{t}^{-1}\right) \\
= & X_{-r}\left(2 t^{-1}\right) h n_{r} n_{r} n_{r} X_{-r}\left(2 t^{-1}\right)
\end{aligned}
$$

where $h=h_{-r}\left(t^{-1}\right) h_{-s}\left(2 k_{r}^{-1} \bar{t}^{-1}\right) h_{-r}\left(t^{-1}\right)$.

Since $X_{r}^{1}(t), X_{-r}^{1}\left(2 t^{-1}\right) \in G^{1}$, we have $h n_{r} n_{r} n_{r} \in G^{1}$. It is easily verified that $h=h_{-s}^{1}\left(\tilde{t}^{-1}\right)$, so $h_{-s}^{1}\left(\tilde{t}^{-1}\right) n_{r} n_{r} n_{r} \in G^{1}$ where $\tilde{t}=\bar{t} t k_{r}$.

By the statement mentioned above and [3] we have the following propositions and corollary immediately.

Proposition 4. (1) $H^{1} \subset N^{1} \subset G^{1}$ and $N^{1} / H^{1} \cong W^{1}$.

(2) For any $r \in \Phi^{*}, t \in K_{r}, N_{r}^{1} X_{r}(t) N_{r}^{1}=X_{-r}(u), u \in K_{r}$.

Proposition 5. (a) Let $y \in Y^{1}, h \in H^{1}$ and $n \in N^{1}$. Then $h y h^{-1}, n y n^{-1}$ $\in Y^{1}$.

(b) $H^{1} \subset Y^{1} \subset G^{1}$ and for each $r \in \Phi^{*}, h_{r}^{1}(v) \in H^{1}, v \in K_{r}^{* \prime}$.

(c) Let $r \in \Sigma_{I}^{*}=\Sigma_{I}^{*+} \cup \Sigma_{I}^{*-}, T \in K_{(r)}$ and $y \in Y^{1}$. Then $y X_{r}(T) y^{-1}=$ $X_{r}\left(T^{\prime}\right), T^{\prime} \in K_{(r)}$.

(d) Let $u \in U^{1}, v \in V^{1}$ and $y \in Y^{1}$. Then $y u y^{-1} \in U^{1}$ and $y v y^{-1} \in V^{1}$. 
COROLlary 2. $U^{1} Y^{1}=Y^{1} U^{1}$ and $B^{1}=U^{1} Y^{1}$ is a subgroup of $G^{1}$.

For each $r \in \Delta_{I}^{*}, \Delta_{0}$ is said to be connected with $r$ if there is $\alpha \in \Delta_{0}$ such that $(r, \alpha) \neq 0$. We denote by $\Delta_{r}$ the set $\{r, \bar{r}\}$ if $\Delta_{0}$ is not connected with $r$, and the set $\Delta_{0} \cup\{r, s\}$ if $\Delta_{0}$ is connected with $r$, where $s \in \Delta^{*}$ satisfies $r \neq s$ and $r^{\prime}=s^{\prime}$. All such $\Delta_{r}$ are given in Table II. The following lemma is easily verified.

Lemma 1. (a) Let $r \in \Delta_{l}^{*}$. Then $I_{1}(r)=\left\{s \in \Phi_{I}^{*+} ; W_{r}^{1}(s) \in \Phi^{-}\right\}$.

(b) Let $r \in \Delta_{l}^{*}$ and $b \in B^{1}$. Then $b=X_{r}(T) u^{\prime} y^{\prime}, T \in K_{(r)}, y^{\prime} \in Y^{1}$ and $u^{\prime} \in U^{1}$ satisfy $N_{r}^{1} u^{\prime} N_{r}^{1}=u^{*} \in U^{1}$.

For each $r \in \Delta_{I}^{*}$, let $\Phi_{r}$ denote the root system with the fundamental $\Delta_{r}$ and let $\Phi_{r}^{*}=\Phi_{r} \backslash \Phi_{r} \cap \Phi_{0}$.

Assuming $\Delta_{r} \neq\{r, r\}$, the Satake diagram of $\Delta_{r}$ is given by Case 4 in Table I of [3] (see Table $I_{4}$ of [3]). We shall use the structure constants for each pair of $\Phi_{r}^{*}$ which are determined by Case 4 mentioned above in [3] (see page 21 of [3]). In this note, we write $k+1$ instead of the $n-1$ used in [3] and $p=(k+1)+1$ instead of the $n$ used in [3].

LEMMA 2. Suppose $r \in \Delta_{l}^{*}, I_{1}(r)=\left\{r_{1}, r_{2}, \ldots, r_{k+1}, r_{p}\right\}$ and $T=\left(t_{1}, \ldots, t_{p}\right)$ $\in K_{(r)}, T \neq 0$. Then $X_{-r}(T)=X_{r}\left(T^{*}\right) N_{r}^{1} X_{r}\left(T^{* \prime}\right) y, y \in Y^{1}, T^{*}, T^{* \prime} \in K_{(r)}$.

Proof. We shall consider the Cases 1, 2 and 3.

(I) Cases 1 and 2 are just the Cases 1 and 2 of Lemma 1 of [3]. Thus, by the results of Lemma 1 of [3], our lemma is established immediately for these Cases 1 and 2.

(II) Case 3 is just the Case 4 of Lemma 1 of [3]. We shall use the notations used in the Case 4 of Lemma 1 of [3]. By the statement (A), we have $k_{-r_{i}}=$ $\gamma_{i}^{-1}$ for all $i=1,2, \ldots, k+1$. Since $T \neq 0$, there is an integer $m, 1 \leq m \leq$ $k+1$, such that $t_{k+1}=\cdots=t_{m+1}=0$ and $t_{m} \neq 0$. We write $\bar{t}=-\gamma^{-1} \bar{t}_{m}$ and $r_{p}=r_{0}$, and $t_{0}=\frac{1}{2} \eta D(T)+\varepsilon, \varepsilon \in K_{r_{0}}, \bar{\varepsilon}=-\varepsilon, D(T)=\sum_{i=1}^{k+1} \gamma_{i}^{-1} t_{i} \bar{t}_{i}$. As in the Case 4 of Lemma 1 of [3] we have

$$
\begin{aligned}
X_{-r}(T)= & X_{-r_{0}}^{1}\left(t_{0}\right) X_{-r_{1}}^{1}\left(t_{1}\right) \cdots X_{-r_{k+1}}^{1}\left(t_{k+1}\right) \\
= & x_{-r_{m}}(\bar{t}) x_{-r_{m}}\left(t_{m}\right) x_{-r_{0}}\left(t_{0}\right) x_{-r_{1}}\left(-\gamma_{1}^{-1} \bar{t}_{1}\right) x_{-r_{1}}\left(t_{1}\right) \\
& \cdots x_{-r_{m-1}}\left(-\gamma_{m-1}^{-1} \bar{t}_{m-1}\right) x_{-r_{m-1}}\left(t_{m-1}\right) \\
= & x_{-r_{m}}\left(\bar{t}^{-1}\right) h_{r_{m}}\left(-\bar{t}^{-1}\right) n_{r_{m}} x_{-r_{m}}\left(\bar{t}^{-1} t_{0}^{*}\right) x_{-r_{0}}\left(t_{0}\right) \\
& x_{-r_{1}}\left(-\gamma_{1}^{-1} t_{1}\right) x_{-r_{1}}\left(t_{1}\right) \cdots x_{-r_{m-1}}\left(-\gamma_{m-1}^{-1} \bar{t}_{m-1}\right) x_{-r_{m-1}}\left(t_{m-1}\right) x_{r_{m}}\left(\bar{t}^{-1}\right) y_{1},
\end{aligned}
$$

where

$$
y=x_{\beta_{1}}\left(\gamma_{1}^{-1} \xi_{1} \bar{t}^{-1} \bar{t}_{1}\right) \cdots x_{\beta_{m-1}}\left(\gamma_{m-1}^{-1} \xi_{m-1} \bar{t}^{-1} \bar{t}_{m-1}\right)
$$


and

$$
t_{0}^{*}=\bar{t}^{-1}\left(\frac{1}{2} D(T)+\eta \varepsilon\right) .
$$

Since $\bar{\gamma}_{i}=\gamma_{i}$, an $\gamma_{i} \neq 0$ for all $i=1,2, \ldots, k+1$ and $T \neq 0$ we have $D(T)=\sum_{i=1}^{k+1} \gamma_{i} t_{i}^{*} t_{i}^{*} \neq 0, t_{i}^{*}=\gamma_{i}^{-1} t_{i}, i=1, \ldots, k+1$. Since $\bar{\varepsilon}=-\varepsilon$ and $\overline{D(T)}=D(T)$ we have $t_{0}^{*} \neq 0$. Then, by a similar method to that used in the proof of the Case 4 of Lemma 1 in [3], finally, we have

$$
X_{-r}(T)=X_{r}\left(T^{*}\right) N_{r}^{1} X_{r}\left(T^{* \prime}\right) y, \quad y \in Y^{1}, T^{*}, T^{* \prime} \in K_{(r)}, N_{r}^{1}=n_{r} n_{r} n_{r} .
$$

The lemma is established for Case 3 , and the proof is complete.

LemMA 3. Let $r \in \Delta_{I}^{*}$. Then $B^{1} \cup B^{1} N_{r}^{1} B^{1}$ is a subgroup of $G^{1}$.

Proof. (1) By Proposition 4 and Corollary 2 we have $\left(N_{r}^{1}\right)^{-1}=h N_{r}^{1}$, $h \in H^{1}$ and $B^{1}=\left(B^{1}\right)^{-1}$ respectively. Thus we have

$$
\left(B^{1} N_{r}^{1} B^{1}\right)^{-1}=\left(B^{1}\right)^{-1}\left(N_{r}^{1}\right)^{-1}\left(B^{1}\right)^{-1}=B^{1} N_{r}^{1} B^{1} .
$$

(2) In order to prove that $B^{1} \cup B^{1} N_{r}^{1} B^{1}$ is closed under multiplication it is sufficient to show $N_{r}^{1} B^{1} N_{r}^{1} \subset B^{1} \cup B^{1} N_{r}^{1} B^{1}$. Let $b \in B^{1}$. Then by statement (b) of Lemma 1, we have $x=N_{r}^{1} b N_{r}^{1}=X_{-r}(T) u^{*} y^{*}$ where $T \in$ $K_{(r)}$ and $u^{*}$ is an element of $U^{1}$ as given in statement (b) of Lemma 1, $y^{*}=N_{r}^{1} y^{\prime} N_{r}^{1} \in Y^{1}$. Suppose $T \neq 0$. Then it follows from Lemma 2 that $x=X_{r}\left(T^{*}\right) N_{r}^{1} X_{r}\left(T^{* \prime}\right) y u^{*} y^{*}, T^{*}, T^{* \prime} \in K_{(r)}, y \in Y^{1}$. Thus by Proposition 5 we have $x \in B^{1} N_{r}^{1} B^{1}$. Suppose $T=0$. Then we have $x \in B^{1}$. We may summarize the results considered above in a single formula as follows: $N_{r}^{1} B^{1} N_{r}^{1} \subset B^{1} \cup B^{1} N_{r}^{1} B^{1}$.

It follows from (1) and (2) that $B^{1} \cup B^{1} N_{r}^{1} B^{1}$ is a subgroup of $G^{1}$.

LeMMA 4. Let $r \in \Delta_{l}^{*}$ and $n \in N^{1}$. Then $B^{1} n B^{1} N_{r}^{1} B^{1} \subset B^{1} n N_{r}^{1} B^{1} \cup B^{1} n B^{1}$.

Proof. Let $w$ be the image of $n$ under the natural homomorphism from $N$ onto $W$. Then $w \in W^{1}$. We shall consider two cases separately.

(1) Suppose $w(r) \in \Phi^{+}$. Let $b \in B$ and $x=n b N_{r}^{1}$. Then by statement (b) of Lemma 1, we have $x=n X_{r}(T) u^{\prime} y^{\prime} N_{r}^{1}=n X_{r}(T) n^{-1} n u^{\prime} y^{\prime} N_{r}^{1}=$ $X_{s}\left(T^{\prime}\right) n N_{r}^{1} u^{*} y^{*}$ where $T \in K_{(r)}, T^{\prime} \in K_{(s)}, s=\operatorname{Min} I(w(r))$, and $u^{*}$ is an element of $U^{1}$ as given in statement (b) of Lemma 1 and $y^{*}=N_{r}^{1} y^{\prime} N_{r}^{1} \in Y^{1}$. Clearly, we have $s \in \Sigma_{I}^{*+}$. Thus $X_{s}\left(T^{\prime}\right) \in U^{1} \subset B^{1}$, so $B^{1} n B^{1} N_{r}^{1} B^{1} \subset$ $B^{1} n N_{r}^{1} B^{1}$.

(2) Suppose $w(r) \in \Phi^{-}$. Let $n_{1}=n N_{r}^{1}$ and $w_{1}$ be the image of $n_{1}$ under the natural homomorphism from $N$ onto $W$. Then $w_{1}(r) \in \Phi^{+}$. It follows from statement (1) above that $B^{1} n_{1} B^{1} N_{r}^{1} B^{1} \subset B^{1} n_{1} N_{r}^{1} B^{1}$. Clearly, we have 
$B^{1} n_{1} N_{r}^{1} B^{1} \subset B^{1} n_{1} B^{1} N_{r}^{1} B^{1}$. Thus $B^{1} n_{1} B^{1} N_{r}^{1} B^{1}=B^{1} n_{1} N_{r}^{1} B^{1}=B^{1} n B^{1}$. By Lemma 3 we have

$$
\begin{aligned}
& B^{1} n B^{1} N_{r}^{1} B^{1} \subset B^{1} n_{1} B^{1} N_{r}^{1} B^{1} N_{r}^{1} B^{1} \subset B^{1} n_{1} B^{1}\left(B^{1} \cup B^{1} N_{r}^{1} B^{1}\right) \\
& =B^{1} n N_{r}^{1} B^{1} \cup B^{1} n B^{1} .
\end{aligned}
$$

The proof is complete.

Theorem 1. Let $G_{1}=B^{1} N^{1} B^{1}$. Then $G_{1}$ is a subgroup of $G^{1}$ and $G_{1}=G^{1}$.

Proof. (1) For each $w \in W^{1}$ we have $w^{-1} \in W^{1}$, so $\left(N^{1}\right)^{-1}=N^{1}$ by Proposition 4. It follows that $\left(B^{1} N^{1} B^{1}\right)^{-1}=\left(B^{1}\right)^{-1}\left(N^{1}\right)^{-1}\left(B^{1}\right)^{-1}=$ $B^{1} N^{1} B^{1}$.

(2) In order to prove that $G_{1}$ is closed under multiplication it is sufficient to show $B^{1} n_{1} B^{1} n_{2} B^{1} \subset G^{1}$ for each pair $n_{1}, n_{2} \in N^{1}$. By Propositions 2 and 4 we have $n_{2}=n_{0}^{*} N_{r_{1}}^{1} N_{r_{2}}^{1} \cdots N_{r_{h}}^{1}, r_{i} \in \Delta_{l}^{*}, i=1,2, \ldots, h, n_{0}^{*} \in N^{1}$, the image of $n_{0}^{*}$ under the natural homomorphism from $N$ onto $W$ being an element $w_{0}$ of $W_{0}^{1}$. Thus we have $n_{0}^{*} \in Y^{1} \subset B^{1}$, so we have $B^{1} n_{1} B^{1} n_{2} B^{1} \subset$ $B^{1} n_{1} B^{1} N_{r_{1}}^{1} B^{1} B^{1} N_{r_{2}}^{1} B^{1} \cdots B^{1} N_{r_{h}}^{1} B^{1}, r_{i} \in \Delta_{I}^{*}, i=1,2, \ldots, h$. By Lemma 4 we have

$$
\begin{aligned}
B^{1} n_{1} B^{1} n_{2} B^{1} & \subset\left(B^{1} n_{1} B^{1} \cup B^{1} n_{1} N_{r_{1}}^{1} B^{1}\right) B^{1} N_{r_{2}}^{1} B^{1} \cdots B^{1} N_{r_{h}}^{1} B^{1} \\
& \subset \cdots \subset B^{1} N^{1} B^{1} .
\end{aligned}
$$

By statements (1) and (2) above, $G_{1}$ is a subgroup of $G^{1}$.

Since $B^{1}, N^{1} \subset G_{1}$, we have $U^{1}, V^{1} \subset G_{1}$. It follows that $G_{1}=G^{1}$.

Let $W^{1 *}=W^{1} \backslash W_{0}^{1}$ and $N^{1 *}=\left\langle N_{r}^{1}, r \in \Delta_{I}^{*}\right\rangle$. By Propositions 2 and 4 , for each $w \in W^{1 *}$, we can choose an element $n_{w}$ of $N^{1 *}$ such that $n_{w}$ corresponds to $w$ under the natural homomorphism from $N$ onto $W$. The elements $n_{w}$ for all $w \in W^{1 *}$ form a set $\tilde{N}^{1 *}$. For each $w \in W^{1 *}$, we define $U_{w}^{1,-}=U_{w}^{-} \cap U^{1}$.

Since $\Phi^{*} \cap \Phi_{0}=\varnothing$, by Theorem 1 , we obtain the following corollary immediately. union).

COROLlaRY 3. $G^{1}=\bigcup_{w \in W^{1 *}} B^{1} n_{w} B^{1}=\bigcup_{w \in W^{1 *}} U^{1} Y^{1} n_{w} U_{w}^{1,-}$ (disjoint

This disjoint union gives the decomposition of $G^{1}$ into double cosets with respect to $B^{1}$. This decomposition of $G^{1}$ is called the quasi-Bruhat decomposition of $G^{1}$. Clearly, this quasi-Bruhat decomposition of $G^{1}$ is a generalized Bruhat decomposition of Steinberg groups and Chevalley groups. For each $T \in K_{(r)}, T \neq 0, r \in \Delta_{I}^{*}$, we have $D(T) \neq 0$ since $D(T)$ defined in this note is associated with $\Delta_{0}(f)$. Thus, the group $G^{1}$ constructed in this note has the 
quasi-Bruhat decomposition $G^{1}=B^{1} N^{1} B^{1}$ which is more explicit than the quasi-Bruhat decomposition $G^{1}=B^{1} N^{1} V^{1} B^{1}$ (not unique) given in [3] for the group $G^{1}$ constructed in [3].

Henceforth, we assume $K$ is a field of characteristic $p \geq 5$ and assume $\nu(f) \geq 2$ if $f$ is a quadratic form.

By [3], we have the following lemma immediately.

Lemma 5. Let $r, s \in \Phi^{*+}$ such that $r^{\prime} \neq s^{\prime}$. Then there exists $h(\chi) \in H^{1}$ such that $\chi(r)=1, \chi(s) \neq 1$ or $\chi(s)=1, \chi(r) \neq 1$, and $\chi(\beta)=1$ for all $\beta \in \Phi_{0}$.

Corollary 4. Suppose $f$ is a Hermitian form and $r_{1}, r_{2} \in \Phi^{*+}$ such that $r_{1} \neq r_{2}$ and $r_{1} \neq \bar{r}_{2}$. Then there is $h(\chi) \in H^{1}$ such that $\chi\left(r_{1}\right)= \pm 1, \chi\left(r_{2}\right) \neq$ $\chi\left(r_{1}\right)$ or $\chi\left(r_{2}\right)= \pm 1, \chi\left(r_{1}\right) \neq \chi\left(r_{2}\right)$.

CoRollary 5. Suppose $\nu(f) \geq 2$. Then for any $r \in \Phi_{11 b} \cap \Sigma_{I}^{*+}, T \in K_{(r)}$, $T \neq\left(0, \ldots, o, t_{p}\right)$ there are $s_{1} \in \Phi^{*}$ and $u \in K_{s_{1}}^{*}$ such that

$$
x=X_{s_{1}}^{1}(u) X_{r}(T) X_{s_{1}}^{1}(-u)=X_{s}^{1}(v), \quad s \in \Phi_{11 a}, v \in K_{s}^{*} .
$$

Proof. Since $r \in \Phi_{11 b}$ we have $r_{0}=r+\bar{r} \in \Phi_{1 b}$, so $r_{0}=e_{h}-e_{m}$ where $m=n-h$ and $h$ is an integer satisfying $0 \leq h \leq \nu-1$. Then we have $I_{1}(r)=\left\{r_{1}, r_{2}, \ldots, r_{k+1}, r_{p}=r_{0}\right\}$ where $r_{i}=e_{i+\nu-1}-e_{m}, i=1, \ldots, k+1$. Since $T \neq\left(0, \ldots, 0, t_{p}\right)$ there exists at least $t_{j} \in K_{r_{j}}^{*}, 1 \leq j \leq k+1$. Since $\nu(f) \geq 2$ there exists $s_{1}=e_{p}-e_{j+\nu-1} \in \Phi_{11 b}$ where $p$ is an integer satisfying $0 \leq p \leq \nu-1$ and $p \neq h$. Let $u \in K_{s_{1}}^{*}$. Then, by 5.2.2 of [1] we have $X_{s_{1}}^{1}(u) X_{r}(T) X_{s_{1}}^{1}(-u)=X_{s}\left(\lambda t_{j} u\right)$ where $s=e_{p}-e_{m} \in \Phi_{11 a}$ and $\lambda=1$ or -1 . Since $t_{j} \neq 0$ and $u \neq 0$ we have $\lambda t_{j} u \in K_{s}^{*}$, so the corollary follows.

Using a similar method to that used in [3], by Lemma 5 , the following corollary is easily verified.

Corollary 6. Let $n \in N^{1 *}$. Then there exists $h(\chi) \in H^{1}$ such that $n h(\chi) n^{-1}=h\left(\chi^{\prime}\right) \neq h(\chi)$ and $\chi(\beta)=\chi^{\prime}(\beta)=1$ for all $\beta \in \Phi_{0}$.

LemMA 6. Suppose $y \in Y$ and $y \neq I$. Then

(1) $y \notin Z^{1}$ where $Z^{1}$ is the centre of $G^{1}$, and

(2) there exists $u \in U^{1}$ such that $y u y^{-1} u^{-1}=u^{*} \neq I$ or there exists $v \in V^{1}$ such that $y v y^{-1} v^{-1}=v^{*} \neq I$.

Proof. It is clear that statement (1) and statement (2) are equivalent. We shall prove statement (1). 
By a similar method to that used in Lemma 6 of [3], the following statement $(\mathrm{C})$ is easily verified.

(C): Suppose $y \in Z^{1}$. Then $y=h\left(\chi_{0}\right) n_{w}, w \in W_{0}$.

(1) Suppose $y \in Z^{1}$ and $w \neq I$. Then there exists $r \in \Phi_{11 b}$ such that $s \neq r$ and $s \neq r, s=w(r)$. Then we have $X_{r}^{1}(1) y X_{r}^{1}(-1) \neq y$. Thus we have a contradiction.

(2) Suppose $y \in Z^{1}$ and $w=I$. Then by statement (C) we have $y=$ $h\left(\chi_{0}\right) \neq 1$. Thus it follows from (4.B) of [3] that there exists $r \in \Phi^{*}$ such that $\chi_{0}(r) \neq 1$. Then we have $X_{r}^{1}(1) y X_{r}^{1}(-1) \neq y$. Thus we have a contradiction.

By the results of Cases (1) and (2) mentioned above we have $y \notin Z^{1}$.

The proof is complete.

For each $r \in \Phi_{I}^{*+}$, we define $X_{r}^{1}=\left\{X_{r}^{1}(t), t\right.$ runs through $\left.K_{r}\right\}$.

We shall denote by $R^{1}$ an arbitrary normal subgroup of $G^{1}$ satisfying $\left|R^{1}\right|>$ 1.

Using the results mentioned above, by a similar method to that used in [3], the following lemmas, corollary and theorem are easily established.

LEMMA 7. $\left|R^{1} \cap U^{1}\right|>1$.

LEMMA 8. There exists $r \in \Phi_{I}^{*+}$ such that $\left|R^{1} \cap X_{r}^{1}\right|>1$.

LemMa 9. For every $s \in \Phi_{I}^{*+}, X_{s}^{1} \subset R^{1}$.

Corollary 7. $U^{1}, V^{1} \subset R^{1}$.

THEOREM 2. $G^{1}$ is a simple group.

Clearly, $\Delta_{l}^{*}$ can be expressed in the form $\Delta_{I}^{*}=\left\{r_{i}, i \in I\right\}$ where $I$ is a finite set of indices. We shall denote by $n_{i}$ the element $N_{r_{i}}^{1}$ and denote by $w_{i}$ the element $W_{r_{i}}^{1}$ where $i \in I$. We denote by $N_{0}^{1}$ the subgroup of $N^{l}$ consisting of all elements of $N^{1}$ which correspond to the elements of $W_{0}^{1}$ under the natural homomorphism from $N$ onto $W$.

THEOREM 3. The subgroups $B^{1}, N^{1}$ form $a(B, N)$-pair in $G^{1}$.

Proof. We shall verify that the subgroups $B^{1}, N^{1}$ satisfy the axioms BN1BN5 in Section 8.2 of [1].

(1) By Theorem $1, G^{1}$ is generated by $B^{1}$ and $N^{1}$.

(2) It is easily verified that $B^{1} \cap N^{1}=N_{0}^{1}$ and $N_{0}^{1}$ is a normal subgroup of $N^{1}$. 
(3) Clearly, for any $i \in I, n_{i}$ corresponds to $w_{i}$ under the natural homomorphism from $N$ onto $W$. Thus we have $N^{1} /\left(B^{1} \cap N^{1}\right)=N^{1} / N_{0}^{1} \cong W^{1} / W_{0}^{1} \cong$ $W^{1 *}$, where $W^{1 *}$ is generated by $\left\{w_{i}, i \in I\right\}, w_{i}^{2}=I$ for all $i \in I$.

(4) By Lemma 4, for any $n \in N^{1}, i \in I$ we have $B^{1} n_{i} B^{1} n B^{1} \subset B^{1} n_{i} n B^{1} \cup$ $B^{1} n B^{1}$.

(5) By Proposition 4, we have $n_{i} X_{r_{i}}^{1} n_{i}=X_{-r_{i}}^{1}, i \in I$, so $n_{i} B^{1} n_{i} \neq B^{1}$ for all $i \in I$.

The proof is complete.

Let $B_{Z}$ be the set of all linear combinations of the elements of $B$ $=\left\{v_{0}, \ldots, v_{n}\right\}$ with the coefficients in $Z$ of rational integers and let $V=$ $B_{Z} \otimes_{Z} K$. Then $V$ is a vector space of dimension $n+1$ over $K$ and $B \otimes 1=$ $\left\{v_{0} \otimes 1, \ldots, v_{n} \otimes 1\right\}$, abbreviated to $B$, is a basis of $V$. Let $S_{D(h)}$ be the set of all $h \times h$ diagonal matrices over $K$ and $U$ (respectively $W$ ) the set of all upper (respectively lower) unitriangular matrices of $S L_{n+1}(K)$. For each form $f$ with matrix $A$ relative to the basis $B$, we define

$$
\begin{aligned}
& U_{f}=\left\{T \in U ; \bar{T}^{\prime} A T=A\right\} ; \quad V_{f}=\left\{T \in \mathrm{V} ; \bar{T}^{\prime} A T=A\right\} ; \\
& H_{f}=\left\{H \in S_{D(n+1)} ; \bar{H}^{\prime} A H=A\right\}, \\
& Y_{f}=\left\{Y=\left[\begin{array}{ccc}
D_{\nu} & & \\
& Y_{k+1} & \\
& & D_{\nu}^{\times}
\end{array}\right] ; Y_{k+1} \in S L_{k+1}(K), D_{\nu}, D_{\nu}^{\times} \in S_{D(\nu)}, \bar{Y}^{\prime} A Y=A\right\} \text {, } \\
& \mathrm{N}=\left\{n=\left[\begin{array}{ccc}
I_{\nu}-D_{\nu} & & \tilde{D}_{\nu} \\
& I_{k+1} & \\
\tilde{D}_{\nu}^{\prime} & & I_{\nu}-D_{\nu}^{\prime}
\end{array}\right] ; D_{\nu} \in S_{D(\nu)}, D_{\nu}^{2}=D_{\nu}, \tilde{D}_{\nu}=D_{\nu} J_{\nu}\right\}
\end{aligned}
$$

( $I_{h}$ being the $h \times h$ identity matrix).

THEOREM 4. Let $f$ be a Hermitian form or a quadratic form with matrix $A$ (relative to the basis $B$ ). Let $\nu=\nu(f)$ be the Witt index of $f$ and $\Delta_{0}(f)$ be the anisotropic kernel of $f$. Then $A_{n}\left(\nu(f), \Delta_{0}(f) ; K, F\right)=P S U_{n+1}(K, f), \quad F \neq I, F\left(\gamma_{i}\right)=\gamma_{i}, i=1, \ldots, k+1$, if $f$ is a Hermitian form;

$$
A_{n}\left(\nu(f), \Delta_{0}(f) ; K, F\right)=P \Omega_{n+1}(K, f), \quad F=I,
$$

if $f$ is a quadratic form.

Proof. Clearly, we have $\sigma: M \rightarrow-A^{-1} \bar{M}^{\prime} A$ for all $M \in L_{K}$ and it has been shown that $G=A_{n}(K)$ consists of all the automorphisms of $L_{K}$ defined by $M \rightarrow T M T^{-1}, T \in S L_{n+1}(K)$ for all $M \in L_{K}$. We consider which of these automorphisms $T$ commute with $\sigma$. In order for this to be so, $T$ must satisfy the condition $-A^{-1}\left(\bar{T}^{-1}\right)^{\prime} \bar{M}^{\prime} \bar{T}^{\prime} A=-T A^{-1} \bar{M}^{\prime} A T^{-1}$ which implies 
$\bar{M}^{\prime} \bar{T}^{\prime} A T A^{-1}=\bar{T}^{\prime} A T A^{-1} \bar{M}^{\prime}$. As this holds for all $M \in L_{K}$ we must have $\bar{T}^{\prime} A T A^{-1}=\lambda I$ for some $\lambda \in K$. Thus $\bar{T}^{\prime} A T=\lambda A$. Suppose $T$ is an upper unitriangular matrix. Then by comparing the $(0, n)$-coefficients on each side we have $\lambda=1$. Similarly, if $T$ is lower unitriangular a comparison of $(n, 0)$ coefficients shows that $\lambda=1$. Thus the matrices $T$ giving rise to the elements of $U^{1}$ and $V^{1}$ are precisely the matrices of $U_{f}$ and of $V_{f}$ respectively. However, $S U_{n+1}(K, f)$ is generated by its $U_{f}$ and $V_{f}$, and $\Omega_{n+1}(K, f)$ is generated by its $U_{f}$ and $V_{f}$ also (see the Appendix). Thus $G^{1}$, the group generated by $U^{1}$ and $V^{1}$, consists of all transformations $M \rightarrow T M T^{-1}, M \in L_{K}$ where $T \in S U_{n+1}(K, f)$ if $f$ is a Hermitian form, $T \in \Omega_{n+1}(K, f)$ if $f$ is a quadratic form. Therefore, $G^{1}$ is isomorphic to $P S U_{n+1}(K, f)$ if $f$ is a Hermitian form, to $P \Omega_{n+1}(K, f)$ if $f$ is a quadratic form. The proof is complete.

For each form $f$ and each $n$ of $\mathrm{N}$, we define $\left(U_{f}\right)_{n}^{-}=\left\{T \in U_{f} ; n T n^{-1} \in\right.$ $\left.V_{f}\right\}$; and $B_{f}=U_{f} Y_{f}$ and $N_{f}=H_{f} N$. By Theorems 2, 3 and 4 we have the following theorems immediately.

TheOREM 5. (a) $P S U_{n+1}(K, f)=\bigcup_{n \in \mathbb{N}} U_{f} Y_{f} n\left(U_{f}\right)_{n}^{-}, F \neq I$ (disjoint union),

(b) $P \Omega_{n+1}(K, f)=\bigcup_{n \in N} U_{f} Y_{f} n\left(U_{f}\right)_{n}^{-}, F=I$ (disjoint union).

THEOREM 6. (a) $P S U_{n+1}(K, f)$ has a $(B, N)$ pair $\left(B_{f}, N_{f}\right)$.

(b) $P \Omega_{n+1}(K, f)$ has a $(B, N)$ pair $\left(B_{f}, N_{f}\right)$.

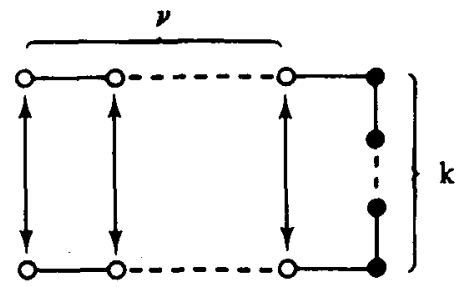

TABLE I

$\mathbf{I}_{1}$

$\begin{array}{llll}0 & I_{2} & 0 & 0 \\ I & & I & \bar{I}\end{array}$

I I3

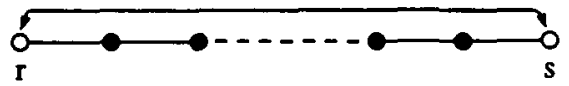

TABLE II

Remark. Suppose $\Delta_{0}=\varnothing$. Then all the nodes of the Dynkin diagram of the " $K$-index" $\left(\Delta, \Delta_{0}, \sigma^{*}\right)$ corresponding to the form $f$ are white, and therefore the group $G^{1}=A_{n}\left(\nu(f), \Delta_{0}(f) ; K, F\right)$ is a Chevalley group or twisted group. Thus $P S U_{n+1}(K, f)$ and $P \Omega_{n+1}(K, f)$ are the orthogonal and unitary groups corresponding to the forms $f$ whose Witt index is sufficiently large. So there is no semi-simple anisotropic kernel for the form $f$, so $Y^{1}=H^{1}$ and $B^{1}=$ $U^{1} H^{1}$, and $B^{1}$ is a Borel subgroup of $G^{1}$. Then $G^{1}$ is $k$-split (Chevalley form) or $k$-quasi-split (Steinberg form). 
Suppose $\Delta_{0} \neq \varnothing$. Then there are some black nodes in the Dynkin diagram of the " $k$-index" $\left(\Delta, \Delta_{0}, \sigma^{*}\right)$ corresponding to the form $f$. Thus $P S U_{n+1}(K, f)$ and $P \Omega_{n+1}(K, f)$ are the orthogonal and unitary groups corresponding to the forms $f$ whose witt index is not sufficiently large. There is a semi-simple anisotropic kernel for the form $f$, so $Y^{1} \neq H^{1}$ and $B^{1} \neq U^{1} H^{1}$, and $B^{1}$ is not a Borel subgroup of $G^{1}$. Then $G^{1}$ is not $k$-split or $k$-quasi-split, so $G^{1}$ is a $k$-non-split form.

\section{Appendix}

In this appendix, we shall prove the following statement.

(A) Suppose $n \geq 5$ and $\nu(f) \geq 1$. Suppose $f$ is a Hermitian form (resp. a quadratic form). Then $P S U_{n+1}(K, f)=\left\langle U_{f}, V_{f}\right\rangle$ (resp. $P \Omega_{n+1}(K, f)=$ $\left.\left\langle U_{f}, V_{f}\right\rangle\right)$.

This statement has been used in the arguments proving Theorem 4.

We first assume that $f$ is a Hermitian form.

We shall denote by $|M|$ the determinant of a matrix $M$. For each basis $B=\left\{v_{0}, \ldots, v_{n}\right\}$ of $V$, there exists an $(n+1) \times(n+1)$ matrix $W$ such that $W\left(v_{i}\right)=v_{i}^{*}, i=0, \ldots, n$, where $v_{i}^{*}=v_{i}, i=0, \ldots, \nu-1, v_{i}^{*}=v_{n+\nu-i}$, $i=\nu, \ldots, 2 \nu-1, v_{i}^{*}=v_{-\nu+i}, i=2 \nu, \ldots, n$. It is easily verified that $B^{*}=$ $\left\{v_{0}^{*}, \ldots, v_{n}^{*}\right\}$ is a basis of $V$ and $\bar{W}^{\prime} W=I$ and

$$
W^{-1} A W=A^{*}=\left[\begin{array}{lll} 
& I_{\nu} & \\
I_{\nu} & & \\
& & A_{0}
\end{array}\right] .
$$

Clearly, the matrix $A$ relative to the basis $B^{*}$ has the form $A^{*}$. Let $f$ be the form with the matrix $A$ relative to the basis $B$. Then $f$ has the form $f^{*}$ with the matrix $A^{*}$ relative to the basis $B^{*}$. Let $f_{1}^{*}$ be the form with the matrix $\left[\begin{array}{c}I_{\nu} \\ I_{\nu}\end{array}\right]$ relative to the basis $B^{*}$. Let $\Delta_{1} \in S_{D(k+1)}$ be such that $\Delta_{1}-\bar{\Delta}_{1}=\Delta$ where $\Delta=A_{0}$. We denote by $S_{P}$ the set of all $\nu \times(k+1)$ matrices over $K$, and denote by $S_{a u}$ (respectively $S_{a l}$ ) the set of all $\nu \times \nu$ upper (respectively lower) unitriangular matrices over $K$. We define

$$
\begin{gathered}
T(C)=\left[\begin{array}{ll}
C & \\
& I_{k+1}
\end{array}\right], \quad C \in U_{2 \nu}\left(K, f_{1}^{*}\right), \\
T\left(C_{1}\right)=\left[\begin{array}{ll}
C_{1} & \\
& I_{k+1}
\end{array}\right], \quad C_{1} \in S U_{2 \nu}\left(K, f_{1}^{*}\right), \\
T\left(H_{1}\right)=\left[\begin{array}{lll}
H_{1} & \bar{H}_{1}^{\prime-1} & \\
& & I_{k+1}
\end{array}\right], \quad H_{1} \in S_{a u},
\end{gathered}
$$


(IIb) $\quad T\left(H_{2}\right)=\left[\begin{array}{lll}H_{2} & & \\ & \bar{H}_{2}^{\prime-1} & \\ & & I_{k+1}\end{array}\right], \quad H_{2} \in S_{a l}$,

(IIIa) $\quad U^{\prime}\left(Q_{1}\right)=\left[\begin{array}{lll}I_{\nu} & Q_{1} & \\ & I_{\nu} & \\ & & I_{k+1}\end{array}\right], \quad Q_{1} \in G L_{\nu}(K), \bar{Q}_{1}^{\prime}=-Q_{1}$,

(IIIb) $U^{2}\left(Q_{2}\right)=\left[\begin{array}{lll}I_{\nu} & & \\ Q_{2} & I_{\nu} & \\ & & I_{k+1}\end{array}\right], \quad Q_{2} \in G L_{\nu}(K), \bar{Q}_{2}^{\prime}=-Q_{2}$,

(IVa)

$$
V^{1}\left(P_{1}\right)=\left[\begin{array}{ccc}
I_{\nu} & -P_{1} \Delta_{1} \bar{P}_{1}^{\prime} & P_{1} \\
& I_{\nu} & \\
& -\Delta \bar{P}_{1}^{\prime} & I_{k+1}
\end{array}\right], \quad P_{1} \in S_{p}
$$

(IVb)

$$
V^{2}\left(P_{2}\right)=\left[\begin{array}{ccc}
I_{\nu} & & \\
-P_{2} \Delta_{1} \bar{P}_{2}^{\prime} & I_{\nu} & P_{2} \\
-\Delta \bar{P}_{2}^{\prime} & & I_{k+1}
\end{array}\right], \quad P_{2} \in S_{p},
$$

(V) $X\left(D_{\nu}\right)=\left[\begin{array}{ccc}D_{\nu} & I_{\nu}-D_{\nu} \\ I_{\nu}-D_{\nu} & D_{\nu} & I_{k+1}\end{array}\right], \quad D_{\nu} \in S_{D(\nu)}, D_{\nu}^{2}=D_{\nu}$,

(VI) $\quad Y(U)=\left[\begin{array}{lll}I_{\nu} & & \\ & I_{\nu} & \\ & & U\end{array}\right], \quad U \in G L_{k+1}(K), \bar{U}^{\prime} A_{0} U=A_{0}$,

(VIs) $Y\left(U_{1}\right)=\left[\begin{array}{lll}I_{\nu} & & \\ & I_{\nu} & \\ & & U_{1}\end{array}\right], \quad U_{1} \in S L_{k+1}(K), \bar{U}_{1}^{\prime} A_{0} U_{1}=A_{0}$.

Henceforth, we assume $n \geq 5,1<\nu \leq \frac{1}{2} n$.

We denote by $G^{*}$ the group generated by all matrices $Y\left(U_{1}\right), U^{1}\left(Q_{1}\right)$, $U^{2}\left(Q_{2}\right), V^{1}\left(P_{1}\right), V^{2}\left(P_{2}\right), T\left(H_{1}\right)$ and $T\left(H_{2}\right)$ mentioned above.

We shall first show the following statements.

(A1) Let $t \in K^{*}$ be such that $t \bar{t}=1$. Then there exists $\lambda \in K^{*}$ such that $t=\lambda \bar{\lambda}^{-1}$.

Proof. Let $\varepsilon$ be a fixed element of $K^{*}$ satisfying $\bar{\varepsilon}=-\varepsilon$ and let $K_{0}=\{t \in$ $K ; \bar{t}=t\}$. Therefore, we have $t=a+b \varepsilon, a, b \in K_{0}$. Since $t \bar{t}=a^{2}-b^{2} \varepsilon^{2}=1$ there exist $x_{0}, y_{0} \in K_{0}$ such that $\lambda=x_{0}+y_{0} \varepsilon \in K^{*}$ and $\left(x_{0}, y_{0}\right)$ satisfies each of the equations in (A.1.1):

$$
(a-1) x-\varepsilon^{2} b y=0, \quad b x-(a+1) y=0
$$

It is easily verified that $\lambda \bar{\lambda}^{-1}=t$. The statement (A1) follows. 


$$
\begin{aligned}
& \text { (1) } X\left(D_{\nu}\right) \in G^{*}, \\
& \text { (2) } 1=\left|X\left(D_{\nu}\right)\right|=|T(Q)|=\left|U^{1}\left(Q_{1}\right)\right| \\
& =\left|U^{2}\left(Q_{2}\right)\right|=\left|V^{1}\left(P_{1}\right)\right|=\left|V^{2}\left(P_{2}\right)\right| .
\end{aligned}
$$

Proof. Since $\left(\begin{array}{cc}0 & 1 \\ -1 & 0\end{array}\right)=\left(\begin{array}{ll}1 & 1 \\ 0 & 1\end{array}\right)\left(\begin{array}{cc}1 & 0 \\ -1 & 1\end{array}\right)\left(\begin{array}{ll}1 & 1 \\ 0 & 1\end{array}\right)$ statement (1) follows. Statement (2) is clear.

The following statement is easily verified.

$$
\begin{aligned}
& Y(U) T(C) Y(U)^{-1}=T(C) ; \quad Y(U) X\left(D_{\nu}\right) Y(U)^{-1}=X\left(D_{\nu}\right) \\
& Y(U) T\left(H_{i}\right) Y(U)^{-1}=T\left(H_{i}\right), \quad i=1,2 \\
& Y(U) U^{i}\left(Q_{i}\right) Y(U)^{-1}=U^{i}\left(Q_{i}\right), \quad i=1,2 \\
& Y(U) V^{i}\left(P_{i}\right) Y(U)^{-1}=V^{i}\left(P_{i} U^{-1}\right), \quad i=1,2 .
\end{aligned}
$$

For each $\lambda \in K^{*}$, we denote by $I_{h}(\lambda)$ the $h \times h$ matrix

$$
\left[\begin{array}{llll}
\lambda_{1} & & & \\
& 1 & & \\
& & \vdots & \\
& & & 1
\end{array}\right]
$$

and we define

$$
I(\lambda)=\left[\begin{array}{lll}
I_{\nu}(\lambda) & & \\
& I_{\nu}\left(\bar{\lambda}^{-1}\right) & \\
& & I_{k+1}
\end{array}\right], \quad D(\lambda)=\left[\begin{array}{lll}
I_{\nu} & & \\
& I_{\nu} & \\
& & I_{k+1}(\lambda)
\end{array}\right]
$$

(A4) Let $T \in S U_{n+1}\left(K, f^{*}\right)$. Then $T$ can be expressed in the form

$$
T=V^{2}\left(P_{2}\right) T\left(C_{1}\right) Y\left(U_{1}\right) V^{1}\left(P_{1}\right) X\left(D_{\nu}\right),
$$

where $T\left(C_{1}\right)$ and $Y\left(U_{1}\right)$ are matrices of types $(\mathrm{Ib})$ and $(\mathrm{VIb})$ respectively.

Proof. By Theorem 3 of Section 8, Chapter 7 of [5], $T$ can be expressed in the form

$$
T=V^{2}\left(P_{2}\right) T(C) Y(U) V^{1}\left(P_{1}\right) X\left(D_{\nu}\right)
$$

where $T(C)$ and $Y(U)$ are matrices of types (I) and (VI) respectively. Let $\mu=|T(U)|$. Then, by (A1), there exists $\lambda \in K^{*}$ such that $\lambda \bar{\lambda}^{-1}=\mu$, so $T$ has the expression $(\mathrm{a}, 4)$ where $T\left(C_{1}\right)=I(\lambda) T(C)$ and $Y\left(U_{1}\right)=D\left(\mu^{-1}\right) Y(U)$. It is easily verified that $T\left(C_{1}\right)$ and $Y\left(U_{1}\right)$ are matrices of type (Is) and (VIs) respectively. The statement (A4) follows.

$$
S U_{n+1}\left(K, f^{*}\right) \subset G^{*}
$$

Proof. By the assertion given in 14.5.1 of [1], all matrices $T\left(C_{1}\right)$ of type (Ib) belong to the subgroup of $G^{*}$ generated by all matrices $T\left(H_{1}\right), T\left(H_{2}\right)$, $U^{1}\left(Q_{1}\right)$ and $U^{2}\left(Q_{2}\right)$. Thus, by (A2) and (A4), statement (A5) follows.

We denote by $\tilde{G}^{*}$ the subgroup of $G$ generated by all $U^{1}\left(Q_{1}\right), U^{2}\left(Q_{2}\right)$, $V^{1}\left(P_{1}\right), V^{2}\left(P_{2}\right), T\left(H_{1}\right)$ and $T\left(H_{2}\right)$ of type (III), (IV) and (II) respectively. 


$$
P S U_{n+1}\left(K, f^{*}\right)=\tilde{G}^{*} \text {. }
$$

Proof. By (A3), $\tilde{G}^{*}$ is a normal subgroup of $G^{*}$. Clearly, all the matrices $T\left(H_{1}\right)$ and $T\left(H_{2}\right)$ of type (II) belong to $P S U_{n+1}\left(K, f^{*}\right)$. By Theorem 1 of Section 2, Chapter 8 of [5], all the matrices $U^{1}\left(Q_{1}\right), U^{2}\left(Q_{2}\right), V^{1}\left(P_{1}\right)$ and $V^{2}\left(P_{2}\right)$ of type (III) and (IV) belong to $P S U_{n+1}\left(K, f^{*}\right)$, so $\tilde{G}^{*}$ is a normal subgroup of $P S U_{n+1}\left(K, f^{*}\right)$. Since $\tilde{G}^{*} \neq\{I\}$ and $P S U_{n+1}\left(K, f^{*}\right)$ is a simple group we have $P S U_{n+1}\left(K, f^{*}\right)=\tilde{G}^{*}$.

(Aa) Let $f$ be a Hermitian form. Then $P S U_{n+1}(K, f)=\left\langle U_{f}, V_{f}\right\rangle$.

Proof. Let $M_{a}$ (respectively $M_{b}$ ) be the set consisting of all the matrices $U^{1}\left(Q_{1}\right), V^{1}\left(P_{1}\right)$ and $T\left(H_{1}\right)$ of type (IIIa), (IVa) and (IIa) (respectively $U^{2}\left(Q_{2}\right), V^{2}\left(P_{2}\right)$ and $T\left(H_{2}\right)$ of type (IIIb), (IVb), and (IIb)). Clearly, all the matrices of $M_{a}$ (respectively of $M_{b}$ ) are upper (respectively lower) unitriangular matrices relative to the basis $B$. Thus, it is easily verified that $U_{f}$ (respectively $V_{f}$ ) coincides with $M_{a}$ (respectively $M_{b}$ ) relative to the basis $B$. By (A6), $P S U_{n+1}\left(K, f^{*}\right)=\left\langle M_{a}, M_{b}\right\rangle$ relative to the basis $B^{*}$, so $P S U_{n+1}(K, f)=\left\langle U_{f}, V_{f}\right\rangle$ relative to the basis $B$. The proof is complete.

We define

$$
J(-1)=\left[\begin{array}{lllll}
0 & & 1 & & \\
& I_{\nu-1} & & & \\
1 & & 0 & & \\
& & & I_{\nu-1} & \\
& & & & I_{k+1}
\end{array}\right]
$$

By a similar argument to that used proving statement (Aa), in which we shall take the matrix $J(-1)$ instead of the matrix $I(\lambda) D\left(\mu^{-1}\right), \lambda, \mu \in K^{*}$, $\lambda \bar{\lambda}^{-1}=\mu$, the following statement (Ab) can be established immediately.

(Ab) Let $f$ be a quadratic form. Then $P \Omega_{n+1}(K, f)=\left\langle U_{f}, V_{f}\right\rangle$.

\section{References}

[1] R. W. Carter, Simple groups of Lie type (Wiley, London, New York, 1972).

[2] Cheng Chon $\mathrm{Hu}$, 'Some simple groups of Lie type constructed by inner automorphism', Chinese Ann. Math. 1 (1980), 161-176.

[3] Cheng Chon $\mathrm{Hu}$, 'A family of simple groups associated with the Satake diagrams', J. Austral. Math. Soc. Ser. A 41 (1986), 13-43.

[4] C. Chevalley, 'Sur certain groups simples', Tôhoku Math. J. 7 (1955), 15-66. 
[5] L. K. Hua and Z. X. Wan, Classical groups (Chinese, Shanghai Ke Ji Press, Shanghai, 1963).

[6] R. Steinberg, 'Variations on a theme of Chevalley', Pacific J. Math. 9 (1959), 875-891.

Department of Mathematics

Xiangtan University

Xiangtan, Hunan Province

People's Republic of China 